\title{
ROTHE TIME-DISCRETIZATION METHOD APPLIED TO A QUASILINEAR WAVE EQUATION SUBJECT TO INTEGRAL CONDITIONS
}

ABDELFATAH BOUZIANI AND NABIL MERAZGA

Received 27 January 2004 and in revised form 12 February 2004

This paper presents a well-posedness result for an initial-boundary value problem with only integral conditions over the spatial domain for a one-dimensional quasilinear wave equation. The solution and some of its properties are obtained by means of a suitable application of the Rothe time-discretization method.

\section{Introduction}

Recently, the study of initial-boundary value problems for hyperbolic equations with boundary integral conditions has received considerable attention. This kind of conditions has many important applications. For instance, they appear in the case where a direct measurement quantity is impossible; however, their mean values are known.

In this paper, we deal with a class of quasilinear hyperbolic equations ( $T$ is a positive constant):

$$
\frac{\partial^{2} v}{\partial t^{2}}-\frac{\partial^{2} v}{\partial x^{2}}=\mathrm{f}\left(x, t, v, \frac{\partial v}{\partial t}\right), \quad(x, t) \in(0,1) \times[0, T]
$$

subject to the initial conditions

$$
v(x, 0)=v_{0}(x), \quad \frac{\partial v}{\partial t}(x, 0)=v_{1}(x), \quad 0 \leqslant x \leqslant 1,
$$

and the boundary integral conditions

$$
\begin{aligned}
& \int_{0}^{1} v(x, t) d x=E(t), \quad 0 \leqslant t \leqslant T, \\
& \int_{0}^{1} x v(x, t) d x=G(t), \quad 0 \leqslant t \leqslant T,
\end{aligned}
$$

where $\mathrm{f}, v_{0}, v_{1}, E$, and $G$ are sufficiently regular given functions.

Problems of this type were first introduced in [3], in which the first author proved the well-posedness of certain linear hyperbolic equations with integral condition(s). Later, 
similar problems have been studied in $[1,4,5,6,7,8,16,24,25]$ by using the energetic method, the Schauder fixed point theorem, Galerkin method, and the theory of characteristics. We refer the reader to $[2,9,10,11,12,13,14,15,17,21,22,23,26]$ for other types of equations with integral conditions.

Differently to these works, in the present paper, we employ the Rothe time-discretization method to construct the solution. This method is a convenient tool for both the theoretical and numerical analyses of the stated problem. Indeed, in addition to giving the first step towards a fully discrete approximation scheme, it provides a constructive proof of the existence of a unique solution. We remark that the application of Rothe method to this nonlocal problem is made possible thanks to the use of the so-called Bouziani space, first introduced by the first author, see, for instance, $[4,6,20]$.

Introducing a new unknown function $u(x, t)=v(x, t)-r(x, t)$, where

$$
r(x, t)=6(2 G(t)-E(t)) x-2(3 G(t)-2 E(t))
$$

problem (1.1)-(1.3) with inhomogeneous integral conditions (1.3) can be equivalently reduced to the problem of finding a function $u$ satisfying

$$
\begin{gathered}
\frac{\partial^{2} u}{\partial t^{2}}-\frac{\partial^{2} u}{\partial x^{2}}=f\left(x, t, u, \frac{\partial u}{\partial t}\right), \quad(x, t) \in(0,1) \times I, \\
u(x, 0)=U_{0}(x), \quad \frac{\partial u}{\partial t}(x, 0)=U_{1}(x), \quad 0 \leqslant x \leqslant 1, \\
\int_{0}^{1} u(x, t) d x=0, \quad t \in I, \\
\int_{0}^{1} x u(x, t) d x=0, \quad t \in I,
\end{gathered}
$$

where

$$
\begin{gathered}
I:=[0, T] \\
f\left(x, t, u, \frac{\partial u}{\partial t}\right):=\mathrm{f}\left(x, t, u+r, \frac{\partial u}{\partial t}+\frac{\partial r}{\partial t}\right)-\frac{\partial^{2} r}{\partial t^{2}} \\
U_{0}(x):=v_{0}(x)-r(x, 0) \\
U_{1}(x)=v_{1}(x)-\frac{\partial r}{\partial t}(x, 0) .
\end{gathered}
$$

Hence, instead of looking for $v$, we simply look for $u$. The solution of problem (1.1)-(1.3) will be directly obtained by the relation $v=u+r$.

The paper is divided as follows. In Section 2, we present notations, definitions, assumptions, and some auxiliary results. Moreover, the concept of the required solution is stated, as well as the main result of the paper. Section 3 is devoted to the construction of approximate solutions of problem (1.5)-(1.8) by solving the corresponding linearized time-discretized problems, while in Section 4, some a priori estimates for the approximations are derived. We end the paper by Section 5 where we prove the convergence of the method and the well-posedness of the investigated problem. 


\section{Preliminaries, notation, and main result}

Let $H^{2}(0,1)$ be the (real) second-order Sobolev space on $(0,1)$ with norm $\|\cdot\|_{H^{2}(0,1)}$ and let $(\cdot, \cdot)$ and $\|\cdot\|$ be the usual inner product and the corresponding norm, respectively, in $L^{2}(0,1)$. The nature of the boundary conditions (1.7) and (1.8) suggests introducing the following space:

$$
V:=\left\{\phi \in L^{2}(0,1) ; \int_{0}^{1} \phi(x) d x=\int_{0}^{1} x \phi(x) d x=0\right\},
$$

which is clearly a Hilbert space for $(\cdot, \cdot)$.

Our analysis requires the use of the so-called Bouziani space $B_{2}^{1}(0,1)$ (see, e.g., $[4,5]$ ) defined as the completion of the space $C_{0}(0,1)$ of real continuous functions with compact support in $(0,1)$, for the inner product

$$
(u, v)_{B_{2}^{1}}=\int_{0}^{1} \mathfrak{J}_{x} u \cdot \mathfrak{J}_{x} v d x
$$

and the associated norm

$$
\|v\|_{B_{2}^{1}}=\sqrt{(v, v)_{B_{2}^{1}}}
$$

where $\mathfrak{J}_{x} v:=\int_{0}^{x} v(\xi) d \xi$ for every fixed $x \in(0,1)$. We recall that, for every $v \in L^{2}(0,1)$, the inequality

$$
\|v\|_{B_{2}^{1}}^{2} \leqslant \frac{1}{2}\|v\|^{2}
$$

holds, implying the continuity of the embedding $L^{2}(0,1) \hookrightarrow B_{2}^{1}(0,1)$.

Moreover, we will work in the standard functional spaces of the types $C(I, X)$, $C^{0,1}(I, X), L^{2}(I, X)$, and $L^{\infty}(I, X)$, where $X$ is a Banach space, the main properties of which can be found in [19].

For a given function $w(x, t)$, the notation $w(t)$ is automatically used for the same function considered as an abstract function of the variable $t \in I$ into some functional space on $(0,1)$. Strong or weak convergence is denoted by $\rightarrow$ or $\rightarrow$, respectively.

The Gronwall lemma in the following continuous and discrete forms will be very useful to us thereafter.

Lemma 2.1. (i) Let $x(t) \geqslant 0$, and let $h(t), y(t)$ be real integrable functions on the interval $[a, b]$. If

$$
y(t) \leqslant h(t)+\int_{a}^{t} x(\tau) y(\tau) d \tau, \quad \forall t \in[a, b]
$$

then

$$
y(t) \leqslant h(t)+\int_{a}^{t} h(\tau) x(\tau) \exp \left(\int_{\tau}^{t} x(s) d s\right) d \tau, \quad \forall t \in[a, b]
$$


214 On a quasilinear wave equation with integral conditions

In particular, if $x(\tau) \equiv C$ is a constant and $h(\tau)$ is nondecreasing, then

$$
y(t) \leqslant h(t) e^{C(t-a)}, \quad \forall t \in[a, b] .
$$

(ii) Let $\left\{a_{i}\right\}$ be a sequence of real nonnegative numbers satisfying

$$
a_{i} \leqslant a+b h \sum_{k=1}^{i} a_{k}, \quad \forall i=1, \ldots
$$

where $a, b$, and $h$ are positive constants with $h<1 / b$. Then

$$
a_{i} \leqslant \frac{a}{1-b h} \exp \left(\frac{b(i-1) h}{1-b h}\right), \quad \forall i=1,2, \ldots
$$

Proof. The proof is the same as that of [18, Lemma 1.3.19].

Throughout the paper, we will make the following assumptions:

$\left(\mathrm{H}_{1}\right) f(t, w, p) \in L^{2}(0,1)$ for each $(t, w, p) \in I \times V \times V$ and the following Lipschitz condition:

$$
\left\|f(t, w, p)-f\left(t^{\prime}, w^{\prime}, p^{\prime}\right)\right\|_{B_{2}^{1}} \leqslant l\left(\left|t-t^{\prime}\right|+\left\|w-w^{\prime}\right\|_{B_{2}^{1}}+\left\|p-p^{\prime}\right\|_{B_{2}^{1}}\right)
$$

is satisfied for all $t, t^{\prime} \in I$ and all $w, w^{\prime}, p, p^{\prime} \in V$, for some positive constant $l$;

$\left(\mathrm{H}_{2}\right) U_{0}, U_{1} \in H^{2}(0,1)$;

$\left(\mathrm{H}_{3}\right)$ the compatibility condition $U_{0}, U_{1} \in V$, that is, concretely,

$$
\begin{aligned}
& \int_{0}^{1} U_{0}(x) d x=\int_{0}^{1} x U_{0}(x) d x=0 \\
& \int_{0}^{1} U_{1}(x) d x=\int_{0}^{1} x U_{1}(x) d x=0 .
\end{aligned}
$$

We look for a weak solution in the following sense.

Definition 2.2. A weak solution of problem (1.5)-(1.8) means a function $u: I \rightarrow L^{2}(0,1)$ such that

(i) $u \in C^{0,1}(I, V)$;

(ii) $u$ has (a.e. in $I$ ) strong derivatives $d u / d t \in L^{\infty}(I, V) \cap C^{0,1}\left(I, B_{2}^{1}(0,1)\right)$ and $d^{2} u / d t^{2} \in L^{\infty}\left(I, B_{2}^{1}(0,1)\right)$

(iii) $u(0)=U_{0}$ in $V$ and $(d u / d t)(0)=U_{1}$ in $B_{2}^{1}(0,1)$;

(iv) the identity

$$
\left(\frac{d^{2} u}{d t^{2}}(t), \phi\right)_{B_{2}^{1}}+(u(t), \phi)=\left(f\left(t, u(t), \frac{d u}{d t}(t)\right), \phi\right)_{B_{2}^{1}}
$$

holds for all $\phi \in V$ and a.e. $t \in I$. 
Note that since $u \in C^{0,1}(I, V)$ and $d u / d t \in C^{0,1}\left(I, B_{2}^{1}(0,1)\right)$, condition (iii) makes sense, whereas assumption $\left(\mathrm{H}_{1}\right)$, together with (i) and the fact that $d u / d t \in L^{\infty}(I, V)$ and $d^{2} u / d t^{2} \in L^{\infty}\left(I, B_{2}^{1}(0,1)\right)$, implies that (2.13) is well defined. On the other hand, the fulfillment of the integral conditions (1.7) and (1.8) is included in the fact that $u(t) \in V$, for all $t \in I$.

The main result of the present paper reads as follows.

Theorem 2.3. Under assumptions $\left(H_{1}\right),\left(H_{2}\right)$, and $\left(H_{3}\right)$, problem (1.5)-(1.8) admits a unique weak solution $u$, in the sense of Definition 2.2, that depends continuously upon the data $f, U_{0}$, and $U_{1}$. Moreover, the following convergence statements hold:

$$
\begin{gathered}
u^{n} \longrightarrow u \quad \text { in } C(I, V), \quad \text { with convergence order } O\left(\frac{1}{n^{1 / 2}}\right), \\
\delta u^{n} \longrightarrow \frac{d u}{d t} \quad \text { in } C\left(I, B_{2}^{1}(0,1)\right), \\
\frac{d}{d t} \delta u^{n} \rightarrow \frac{d^{2} u}{d t^{2}} \quad \text { in } L^{2}\left(I, B_{2}^{1}(0,1)\right),
\end{gathered}
$$

as $n \rightarrow \infty$, where the sequences $\left\{u^{n}\right\}_{n}$ and $\left\{\delta u^{n}\right\}_{n}$ are defined in (3.18) and (3.19), respectively.

\section{Construction of an approximate solution}

Let $n$ be an arbitrary positive integer, and let $\left\{t_{j}\right\}_{j=1}^{n}$ be the uniform partition of $I, t_{j}=j h_{n}$ with $h_{n}=T / n$. Successively, for $j=1, \ldots, n$, we solve the linear stationary boundary value problem

$$
\begin{aligned}
\frac{u_{j}-2 u_{j-1}+u_{j-2}}{h_{n}^{2}}-\frac{d^{2} u_{j}}{d x^{2}} & =f_{j}, \quad x \in(0,1), \\
\int_{0}^{1} u_{j}(x) d x & =0 \\
\int_{0}^{1} x u_{j}(x) d x & =0
\end{aligned}
$$

where

$$
f_{j}:=f\left(t_{j}, u_{j-1}, \frac{u_{j-1}-u_{j-2}}{h_{n}}\right)
$$

starting from

$$
u_{-1}(x)=U_{0}(x)-h_{n} U_{1}(x), \quad u_{0}(x)=U_{0}(x), \quad x \in(0,1) .
$$

Lemma 3.1. For each $n \in \mathbb{N}^{*}$ and each $j=1, \ldots, n$, problem $(3.1)_{j}-(3.3)_{j}$ admits a unique solution $u_{j} \in H^{2}(0,1)$.

Proof. We use induction on $j$. For this, suppose that $u_{j-1}$ and $u_{j-2}$ are already known and that they belong to $H^{2}(0,1)$, then $f_{j} \in L^{2}(0,1)$. From the classical theory of linear ordinary differential equations with constant coefficients, the general solution of $(3.1)_{j}$ 
216 On a quasilinear wave equation with integral conditions

which can be written in the form

$$
\frac{d^{2} u_{j}}{d x^{2}}-\frac{1}{h_{n}^{2}} u_{j}=\frac{-2 u_{j-1}+u_{j-2}}{h_{n}^{2}}-f_{j}
$$

is given by

$$
u_{j}(x)=k_{1}(x) \cosh \frac{x}{h_{n}}+k_{2}(x) \sinh \frac{x}{h_{n}}, \quad x \in(0,1),
$$

where $k_{1}$ and $k_{2}$ are two functions of $x$ satisfying the linear algebraic system

$$
\begin{gathered}
\frac{d k_{1}}{d x}(x) \cosh \frac{x}{h_{n}}+\frac{d k_{2}}{d x}(x) \sinh \frac{x}{h_{n}}=0, \\
\frac{d k_{1}}{d x}(x) \sinh \frac{x}{h_{n}}+\frac{d k_{2}}{d x}(x) \cosh \frac{x}{h_{n}}=h_{n} F_{j}(x),
\end{gathered}
$$

with

$$
F_{j}:=\frac{-2 u_{j-1}+u_{j-2}}{h_{n}^{2}}-f_{j} .
$$

Since the determinant of (3.8) is

$$
\Delta=\cosh ^{2} \frac{x}{h_{n}}-\sinh ^{2} \frac{x}{h_{n}}=1,
$$

then

$$
\begin{aligned}
& \frac{d k_{1}}{d x}(x)=\left|\begin{array}{cc}
0 & \sinh \frac{x}{h_{n}} \\
h_{n} F_{j}(x) & \cosh \frac{x}{h_{n}}
\end{array}\right|=-h_{n} F_{j}(x) \sinh \frac{x}{h_{n}}, \\
& \frac{d k_{2}}{d x}(x)=\left|\begin{array}{cc}
\cosh \frac{x}{h_{n}} & 0 \\
\sinh \frac{x}{h_{n}} & h_{n} F_{j}(x)
\end{array}\right|=h_{n} F_{j}(x) \cosh \frac{x}{h_{n}},
\end{aligned}
$$

that is,

$$
\begin{aligned}
& k_{1}(x)=-h_{n} \int_{0}^{x} F_{j}(\xi) \sinh \frac{\xi}{h_{n}} d \xi+\lambda_{1}, \\
& k_{2}(x)=h_{n} \int_{0}^{x} F_{j}(\xi) \cosh \frac{\xi}{h_{n}} d \xi+\lambda_{2},
\end{aligned}
$$

with $\lambda_{1}$ and $\lambda_{2}$ two arbitrary real constants. Inserting (3.12) into (3.7), we get

$$
u_{j}(x)=h_{n} \int_{0}^{x} F_{j}(\xi) \sinh \frac{x-\xi}{h_{n}} d \xi+\lambda_{1} \cosh \frac{x}{h_{n}}+\lambda_{2} \sinh \frac{x}{h_{n}} .
$$


Obviously, the function $u_{j}$ will be a solution to problem $(3.1)_{j}-(3.3)_{j}$ if and only if the pair $\left(\lambda_{1}, \lambda_{2}\right)$ is selected in such a manner that conditions $(3.2)_{j}$ and $(3.3)_{j}$ hold, that is,

$$
\begin{gathered}
\lambda_{1} \int_{0}^{1} \cosh \frac{x}{h_{n}} d x+\lambda_{2} \int_{0}^{1} \sinh \frac{x}{h_{n}} d x=-h_{n} \int_{0}^{1} \int_{0}^{x} F_{j}(\xi) \sinh \frac{x-\xi}{h_{n}} d \xi d x, \\
\lambda_{1} \int_{0}^{1} x \cosh \frac{x}{h_{n}} d x+\lambda_{2} \int_{0}^{1} x \sinh \frac{x}{h_{n}} d x=-h_{n} \int_{0}^{1} \int_{0}^{x} x F_{j}(\xi) \sinh \frac{x-\xi}{h_{n}} d \xi d x .
\end{gathered}
$$

An easy computation shows that $\left(\lambda_{1}, \lambda_{2}\right)$ is the solution of the linear algebraic system

$$
\begin{gathered}
\lambda_{1} \sinh \frac{1}{h_{n}}+\lambda_{2}\left(\cosh \frac{1}{h_{n}}-1\right)=-\int_{0}^{1} \int_{0}^{x} F_{j}(\xi) \sinh \frac{x-\xi}{h_{n}} d \xi d x, \\
\lambda_{1}\left(\sinh \frac{1}{h_{n}}-h_{n} \cosh \frac{1}{h_{n}}+h_{n}\right)+\lambda_{2}\left(\cosh \frac{1}{h_{n}}-h_{n} \sinh \frac{1}{h_{n}}\right) \\
=-\int_{0}^{1} \int_{0}^{x} x F_{j}(\xi) \sinh \frac{x-\xi}{h_{n}} d \xi d x,
\end{gathered}
$$

whose determinant is

$$
\begin{aligned}
D\left(h_{n}\right) & =2 h_{n}-2 h_{n} \cosh \frac{1}{h_{n}}+\sinh \frac{1}{h_{n}} \\
& =2 \sinh \frac{1}{2 h_{n}}\left(\cosh \frac{1}{2 h_{n}}-2 h_{n} \sinh \frac{1}{2 h_{n}}\right) .
\end{aligned}
$$

Note that $D\left(h_{n}\right)$ does not vanish for any $h_{n}>0$, indeed equation $D\left(h_{n}\right)=0$ is equivalent to the equation $\cosh \left(1 / 2 h_{n}\right)-2 h_{n} \sinh \left(1 / 2 h_{n}\right)=0$, that is, to the equation $\tanh \left(1 / 2 h_{n}\right)=$ $1 / 2 h_{n}$ which clearly has no solution. Therefore, for all $h_{n}>0$, system (3.15) admits a unique solution $\left(\lambda_{1}, \lambda_{2}\right) \in \mathbb{R}^{2}$, which means that problem $(3.1)_{j}-(3.3)_{j}$ is uniquely solvable, and it is obvious that $u_{j} \in H^{2}(0,1)$ since $F_{j} \in L^{2}(0,1)$.

Now, we introduce the notations

$$
\begin{gathered}
\delta u_{j}:=\frac{u_{j}-u_{j-1}}{h_{n}}, \quad j=0, \ldots, n, \\
\delta^{2} u_{j}:=\frac{\delta u_{j}-\delta u_{j-1}}{h_{n}}=\frac{u_{j}-2 u_{j-1}+u_{j-2}}{h_{n}^{2}}, \quad j=1, \ldots, n,
\end{gathered}
$$

and construct the Rothe function $u^{n}: I \rightarrow H^{2}(0,1) \cap V$ by setting

$$
u^{n}(t)=u_{j-1}+\delta u_{j}\left(t-t_{j-1}\right), \quad t \in\left[t_{j-1}, t_{j}\right], j=1, \ldots, n,
$$


and the following auxiliary functions:

$$
\begin{gathered}
\delta u^{n}(t)=\delta u_{j-1}+\delta^{2} u_{j}\left(t-t_{j-1}\right), \quad t \in\left[t_{j-1}, t_{j}\right], j=1, \ldots, n, \\
\bar{u}^{n}(t)= \begin{cases}u_{j} & \text { for } t \in\left(t_{j-1}, t_{j}\right], j=1, \ldots, n, \\
U_{0} & \text { for } t \in\left[-h_{n}, 0\right],\end{cases} \\
\overline{\delta u}^{n}(t)= \begin{cases}\delta u_{j} & \text { for } t \in\left(t_{j-1}, t_{j}\right], j=1, \ldots, n, \\
U_{1} & \text { for } t \in\left[-h_{n}, 0\right] .\end{cases}
\end{gathered}
$$

We expect that the limit $u:=\lim _{n \rightarrow \infty} u^{n}$ exists in a suitable sense, and that is the desired weak solution to our problem (1.5)-(1.8). The demonstration of this fact requires some a priori estimates whose derivation is the subject of the following section.

\section{A priori estimates for the approximations}

In what follows, $c$ denote generic positive constants which are not necessarily the same at any two places.

Lemma 4.1. There exist $c>0$ and $n_{0} \in \mathbb{N}^{*}$ such that

$$
\begin{gathered}
\left\|u_{j}\right\| \leqslant c \\
\left\|\delta u_{j}\right\| \leqslant c \\
\left\|\delta^{2} u_{j}\right\|_{B_{2}^{1}} \leqslant c
\end{gathered}
$$

for all $j=1, \ldots, n$ and all $n \geqslant n_{0}$.

Proof. To derive these estimates, we need to write problem $(3.1)_{j}-(3.3)_{j}$ in a weak formulation.

Let $\phi$ be an arbitrary function from the space $V$ defined in (2.1). One can easily find that

$$
\int_{0}^{x}(x-\xi) \phi(\xi) d \xi=\mathfrak{I}_{x}^{2} \phi, \quad \forall x \in(0,1)
$$

where

$$
\mathfrak{J}_{x}^{2} \phi:=\mathfrak{J}_{x}\left(\mathfrak{J}_{\xi} \phi\right)=\int_{0}^{x} d \xi \int_{0}^{\xi} \phi(\eta) d \eta
$$

This implies that

$$
\mathfrak{J}_{1}^{2} \phi=\int_{0}^{1}(1-\xi) \phi(\xi) d \xi=\int_{0}^{1} \phi(\xi) d \xi-\int_{0}^{1} \xi \phi(\xi) d \xi=0 .
$$

Next, we multiply, for all $j=1, \ldots, n,(3.1)_{j}$ by $\mathfrak{J}_{x}^{2} \phi$ and integrate over $(0,1)$ to get

$$
\int_{0}^{1} \delta^{2} u_{j}(x) \mathfrak{I}_{x}^{2} \phi d x-\int_{0}^{1} \frac{d^{2} u_{j}}{d x^{2}}(x) \mathfrak{I}_{x}^{2} \phi d x=\int_{0}^{1} f_{j}(x) \mathfrak{J}_{x}^{2} \phi d x .
$$


Here, we used the notations (3.17). Performing some standard integrations by parts for each term in (4.7) and invoking (4.6), we obtain

$$
\begin{aligned}
\int_{0}^{1} \delta^{2} u_{j}(x) \mathfrak{I}_{x}^{2} \phi d x & =\int_{0}^{1} \frac{d}{d x}\left(\mathfrak{I}_{x}\left(\delta^{2} u_{j}\right)\right) \mathfrak{I}_{x}^{2} \phi d x \\
& =\left.\mathfrak{I}_{x}\left(\delta^{2} u_{j}\right) \mathfrak{I}_{x}^{2} \phi\right|_{x=0} ^{x=1}-\int_{0}^{1} \mathfrak{I}_{x}\left(\delta^{2} u_{j}\right) \mathfrak{I}_{x} \phi d x \\
& =-\left(\delta^{2} u_{j}, \phi\right)_{B_{2}^{1}}, \quad \\
\int_{0}^{1} \frac{d^{2} u_{j}}{d x^{2}}(x) \mathfrak{I}_{x}^{2} \phi d x & =\left.\frac{d u_{j}}{d x}(x) \mathfrak{I}_{x}^{2} \phi\right|_{x=0} ^{x=1}-\int_{0}^{1} \frac{d u_{j}}{d x}(x) \mathfrak{I}_{x} \phi d x \\
& =-\int_{0}^{1} \frac{d u_{j}}{d x}(x) \mathfrak{I}_{x} \phi d x \\
& =-\left.u_{j}(x) \mathfrak{I}_{x} \phi\right|_{x=0} ^{x=1}+\int_{0}^{1} u_{j}(x) \phi(x) d x \\
& =\left(u_{j}, \phi\right), \\
\int_{0}^{1} f_{j}(x) \mathfrak{I}_{x}^{2} \phi d x & =\int_{0}^{1} \frac{d}{d x}\left(\mathfrak{I}_{x} f_{j}\right) \mathfrak{I}_{x}^{2} \phi d x \\
& =\left.\mathfrak{I}_{x} f_{j} \mathfrak{I}_{x}^{2} \phi\right|_{x=0} ^{x=1}-\int_{0}^{1} \mathfrak{I}_{x} f_{j} \mathfrak{I}_{x} \phi d x \\
& =-\left(f_{j}, \phi\right)_{B_{2}^{1}},
\end{aligned}
$$

so that (4.7) becomes finally

$$
\left(\delta^{2} u_{j}, \phi\right)_{B_{2}^{1}}+\left(u_{j}, \phi\right)=\left(f_{j}, \phi\right)_{B_{2}^{1}}, \quad \forall \phi \in V, \forall j=1, \ldots, n
$$

Now, for $i=2, \ldots, j$, we take the difference of the relations $(4.9)_{i}-(4.9)_{i-1}$, tested with $\phi=\delta^{2} u_{i}=\left(\delta u_{i}-\delta u_{i-1}\right) / h_{n}$ which belongs to $V$ in view of $(3.2)_{i}-(3.3)_{i},(3.2)_{i-1}-$ $(3.3)_{i-1}$, and $\left(\mathrm{H}_{3}\right)$. We have

$$
\left(\delta^{2} u_{i}-\delta^{2} u_{i-1}, \delta^{2} u_{i}\right)_{B_{2}^{1}}+\left(\delta u_{i}, \delta u_{i}-\delta u_{i-1}\right)=\left(f_{i}-f_{i-1}, \delta^{2} u_{i}\right)_{B_{2}^{1}},
$$

then, using the identity

$$
2(v, v-w)=\|v\|^{2}-\|w\|^{2}+\|v-w\|^{2}
$$

and its analog for $(\cdot, \cdot)_{B_{2}^{1}}$, it follows that

$$
\begin{aligned}
\left\|\delta^{2} u_{i}\right\|_{B_{2}^{1}}^{2}-\left\|\delta^{2} u_{i-1}\right\|_{B_{2}^{1}}^{2}+\left\|\delta^{2} u_{i}-\delta^{2} u_{i-1}\right\|_{B_{2}^{1}}^{2}+\left\|\delta u_{i}\right\|^{2} \\
\quad-\left\|\delta u_{i-1}\right\|^{2}+\left\|\delta u_{i}-\delta u_{i-1}\right\|^{2}=2\left(f_{i}-f_{i-1}, \delta^{2} u_{i}\right)_{B_{2}^{1}},
\end{aligned}
$$

hence, omitting the third and last terms in the left-hand side, we get

$$
\left\|\delta^{2} u_{i}\right\|_{B_{2}^{1}}^{2}+\left\|\delta u_{i}\right\|^{2} \leqslant\left\|\delta^{2} u_{i-1}\right\|_{B_{2}^{1}}^{2}+\left\|\delta u_{i-1}\right\|^{2}+\left.2\left\|f_{i}-f_{i-1}\right\|\right|_{B_{2}^{1}}\left\|\delta^{2} u_{i}\right\|_{B_{2}^{1}} .
$$


We sum up these inequalities and obtain

$$
\left\|\delta^{2} u_{j}\right\|_{B_{2}^{1}}^{2}+\left\|\delta u_{j}\right\|^{2} \leqslant\left\|\delta^{2} u_{1}\right\|_{B_{2}^{1}}^{2}+\left\|\delta u_{1}\right\|^{2}+2 \sum_{i=2}^{j}\left\|f_{i}-f_{i-1}\right\|_{B_{2}^{1}}\left\|\delta^{2} u_{i}\right\|_{B_{2}^{1}}
$$

hence, thanks to the Cauchy inequality

$$
2 a b \leqslant \frac{1}{\varepsilon} a^{2}+\varepsilon b^{2}, \quad \forall a, b \in \mathbb{R}, \forall \varepsilon \in \mathbb{R}_{+}^{*},
$$

we can write, for $\varepsilon=h_{n}$,

$$
\left\|\delta^{2} u_{j}\right\|_{B_{2}^{1}}^{2}+\left\|\delta u_{j}\right\|^{2} \leqslant\left\|\delta^{2} u_{1}\right\|_{B_{2}^{1}}^{2}+\left\|\delta u_{1}\right\|^{2}+\frac{1}{h_{n}} \sum_{i=2}^{j}\left\|f_{i}-f_{i-1}\right\|_{B_{2}^{1}}^{2}+h_{n} \sum_{i=2}^{j}\left\|\delta^{2} u_{i}\right\|_{B_{2}^{1}}^{2}
$$

To majorize $\sum_{i=2}^{j}\left\|f_{i}-f_{i-1}\right\|_{B_{2}^{1}}^{2}$, we remark that

$$
\begin{aligned}
\left\|f_{i}-f_{i-1}\right\|_{B_{2}^{1}}^{2} & =\left\|f\left(t_{i}, u_{i-1}, \delta u_{i-1}\right)-f\left(t_{i-1}, u_{i-2}, \delta u_{i-2}\right)\right\|_{B_{2}^{1}}^{2} \\
& \leqslant l^{2}\left(h_{n}+\left\|u_{i-1}-u_{i-2}\right\|_{B_{2}^{1}}+\left\|\delta u_{i-1}-\delta u_{i-2}\right\|_{B_{2}^{1}}\right)^{2} \\
& =l^{2} h_{n}^{2}\left(1+\left\|\delta u_{i-1}\right\|_{B_{2}^{1}}+\left\|\delta^{2} u_{i-1}\right\|_{B_{2}^{1}}\right)^{2} \\
& \leqslant 3 l^{2} h_{n}^{2}\left(1+\left\|\delta u_{i-1}\right\|_{B_{2}^{1}}^{2}+\left\|\delta^{2} u_{i-1}\right\|_{B_{2}^{1}}^{2}\right), \quad i=2, \ldots, j .
\end{aligned}
$$

Summing up for $i=2, \ldots, j$, we may arrive at

$$
\sum_{i=2}^{j}\left\|f_{i}-f_{i-1}\right\|_{B_{2}^{1}}^{2} \leqslant 3 l^{2}(j-1) h_{n}^{2}+3 l^{2} h_{n}^{2} \sum_{i=2}^{j}\left(\left\|\delta u_{i-1}\right\|_{B_{2}^{1}}^{2}+\left\|\delta^{2} u_{i-1}\right\|_{B_{2}^{1}}^{2}\right)
$$

or

$$
\sum_{i=2}^{j}\left\|f_{i}-f_{i-1}\right\|_{B_{2}^{1}}^{2} \leqslant 3 l^{2}(j-1) h_{n}^{2}+3 l^{2} h_{n}^{2} \sum_{i=1}^{j-1}\left(\left\|\delta^{2} u_{i}\right\|_{B_{2}^{1}}^{2}+\left\|\delta u_{i}\right\|_{B_{2}^{1}}^{2}\right) .
$$

To estimate $\left\|\delta^{2} u_{1}\right\|_{B_{2}^{1}}^{2}+\left\|\delta u_{1}\right\|^{2}$, we test the relation (4.9) $)_{1}$ with $\phi=\delta^{2} u_{1}=\left(\delta u_{1}-\delta u_{0}\right) / h_{n}$ $=\left(\delta u_{1}-U_{1}\right) / h_{n}$ which is an element of $V$ owing to $(3.2)_{1}-(3.3)_{1}$ and assumption $\left(\mathrm{H}_{3}\right)$. We have

$$
\left\|\delta^{2} u_{1}\right\|_{B_{2}^{1}}^{2}+\left(\frac{u_{1}}{h_{n}}, \delta u_{1}-U_{1}\right)=\left(f_{1}, \delta^{2} u_{1}\right)_{B_{2}^{1}}
$$

or

$$
\left\|\delta^{2} u_{1}\right\|_{B_{2}^{1}}^{2}+\left(\delta u_{1}, \delta u_{1}-U_{1}\right)=\left(f_{1}, \delta^{2} u_{1}\right)_{B_{2}^{1}}-\left(U_{0}, \delta^{2} u_{1}\right)
$$


But

$$
\begin{aligned}
\left(U_{0}, \delta^{2} u_{1}\right) & =\int_{0}^{1} U_{0}(x) \frac{d}{d x}\left(\mathfrak{I}_{x} \delta^{2} u_{1}\right) d x \\
& =\left.U_{0}(x) \mathfrak{I}_{x} \delta^{2} u_{1}\right|_{x=0} ^{x=1}-\int_{0}^{1} \frac{d U_{0}}{d x}(x) \mathfrak{I}_{x} \delta^{2} u_{1} d x \\
& =-\int_{0}^{1} \frac{d U_{0}}{d x}(x) \mathfrak{I}_{x} \delta^{2} u_{1} d x
\end{aligned}
$$

and since

$$
\mathfrak{J}_{x}\left(\frac{d^{2} U_{0}}{d x^{2}}\right)=\frac{d U_{0}}{d x}(x)-\frac{d U_{0}}{d x}(0), \quad \forall x \in(0,1),
$$

we get, due to (4.6),

$$
\begin{aligned}
\left(U_{0}, \delta^{2} u_{1}\right) & =-\int_{0}^{1} \mathfrak{I}_{x}\left(\frac{d^{2} U_{0}}{d x^{2}}\right) \mathfrak{J}_{x} \delta^{2} u_{1} d x-\frac{d U_{0}}{d x}(0) \mathfrak{I}_{1}^{2} \delta^{2} u_{1} \\
& =-\int_{0}^{1} \mathfrak{I}_{x}\left(\frac{d^{2} U_{0}}{d x^{2}}\right) \mathfrak{J}_{x} \delta^{2} u_{1} d x \\
& =-\left(\frac{d^{2} U_{0}}{d x^{2}}, \delta^{2} u_{1}\right)_{B_{2}^{1}}
\end{aligned}
$$

in light of which (4.21) becomes

$$
\left\|\delta^{2} u_{1}\right\|_{B_{2}^{1}}^{2}+\left(\delta u_{1}, \delta u_{1}-U_{1}\right)=\left(f_{1}+\frac{d^{2} U_{0}}{d x^{2}}, \delta^{2} u_{1}\right)_{B_{2}^{1}}
$$

Therefore,

$$
\left\|\delta^{2} u_{1}\right\|_{B_{2}^{1}}^{2}+\frac{1}{2}\left\|\delta u_{1}\right\|^{2}-\frac{1}{2}\left\|U_{1}\right\|^{2}+\frac{1}{2}\left\|\delta u_{1}-U_{1}\right\|^{2} \leqslant\left\|f_{1}+\frac{d^{2} U_{0}}{d x^{2}}\right\|_{B_{2}^{1}}\left\|\delta^{2} u_{1}\right\|_{B_{2}^{1}},
$$

hence,

$$
\begin{aligned}
2\left\|\delta^{2} u_{1}\right\|_{B_{2}^{1}}^{2}+\left\|\delta u_{1}\right\|^{2} & \leqslant\left\|U_{1}\right\|^{2}+2\left\|f_{1}+\frac{d^{2} U_{0}}{d x^{2}}\right\|_{B_{2}^{1}}\left\|\delta^{2} u_{1}\right\|_{B_{2}^{1}} \\
& \leqslant\left\|U_{1}\right\|^{2}+\left\|f_{1}+\frac{d^{2} U_{0}}{d x^{2}}\right\|_{B_{2}^{1}}^{2}+\left\|\delta^{2} u_{1}\right\|_{B_{2}^{1}}^{2} \\
& \leqslant\left\|U_{1}\right\|^{2}+2\left[\left\|f_{1}\right\|_{B_{2}^{1}}^{2}+\left\|\frac{d^{2} U_{0}}{d x^{2}}\right\|_{B_{2}^{1}}^{2}\right]+\left\|\delta^{2} u_{1}\right\|_{B_{2}^{1}}^{2},
\end{aligned}
$$

from which it follows that

$$
\left\|\delta^{2} u_{1}\right\|_{B_{2}^{1}}^{2}+\left\|\delta u_{1}\right\|^{2} \leqslant\left\|U_{1}\right\|^{2}+2\left[c_{1}+\left\|\frac{d^{2} U_{0}}{d x^{2}}\right\|_{B_{2}^{1}}^{2}\right]
$$


where $c_{1}:=\max _{t \in I}\left\|f\left(t, U_{0}, U_{1}\right)\right\|_{B_{2}^{1}}^{2}<\infty$ in virtue of $\left(H_{1}\right)$. Substituting (4.19) and (4.28) in (4.16), this gives

$$
\begin{aligned}
\left\|\delta^{2} u_{j}\right\|_{B_{2}^{1}}^{2}+\left\|\delta u_{j}\right\|^{2} \leqslant & \left\|U_{1}\right\|^{2}+2\left[c_{1}+\left\|\frac{d^{2} U_{0}}{d x^{2}}\right\|_{B_{2}^{1}}^{2}\right]+3 l^{2}(j-1) h_{n} \\
& +3 l^{2} h_{n} \sum_{i=1}^{j-1}\left(\left\|\delta^{2} u_{i}\right\|_{B_{2}^{1}}^{2}+\left\|\delta u_{i}\right\|_{B_{2}^{1}}^{2}\right)+h_{n} \sum_{i=2}^{j}\left\|\delta^{2} u_{i}\right\|_{B_{2}^{1}}^{2} \\
\leqslant & \left\|U_{1}\right\|^{2}+2\left[c_{1}+\left\|\frac{d^{2} U_{0}}{d x^{2}}\right\|_{B_{2}^{1}}^{2}\right]+3 l^{2}(j-1) h_{n} \\
& +3 l^{2} h_{n} \sum_{i=1}^{j}\left(\left\|\delta^{2} u_{i}\right\|_{B_{2}^{1}}^{2}+\left\|\delta u_{i}\right\|^{2}\right)+h_{n} \sum_{i=1}^{j}\left(\left\|\delta^{2} u_{i}\right\|_{B_{2}^{1}}^{2}+\left\|\delta u_{i}\right\|^{2}\right) \\
= & \left\|U_{1}\right\|^{2}+2\left[c_{1}+\left\|\frac{d^{2} U_{0}}{d x^{2}}\right\|_{B_{2}^{1}}^{2}\right]+3 l^{2}(j-1) h_{n} \\
& +\left(3 l^{2}+1\right) h_{n} \sum_{i=1}^{j}\left(\left\|\delta^{2} u_{i}\right\|_{B_{2}^{1}}^{2}+\left\|\delta u_{i}\right\|^{2}\right) ;
\end{aligned}
$$

consequently,

$$
\begin{aligned}
\left\|\delta^{2} u_{j}\right\|_{B_{2}^{1}}^{2}+\left\|\delta u_{j}\right\|^{2} \leqslant & \left\|U_{1}\right\|^{2}+2\left[c_{1}+\left\|\frac{d^{2} U_{0}}{d x^{2}}\right\|_{B_{2}^{1}}^{2}\right]+3 l^{2} T \\
& +\left(3 l^{2}+1\right) h_{n} \sum_{i=1}^{j}\left(\left\|\delta^{2} u_{i}\right\|_{B_{2}^{1}}^{2}+\left\|\delta u_{i}\right\|^{2}\right), \quad \forall j=1, \ldots, n .
\end{aligned}
$$

By the discrete Gronwall lemma, we conclude that

$$
\begin{aligned}
& \left\|\delta^{2} u_{j}\right\|_{B_{2}^{1}}^{2}+\left\|\delta u_{j}\right\|^{2} \\
& \quad \leqslant \frac{\left\|U_{1}\right\|^{2}+2\left[c_{1}+\left\|d^{2} U_{0} / d x^{2}\right\|_{B_{2}^{1}}^{2}\right]+3 l^{2} T}{1-\left(3 l^{2}+1\right) h_{n}} e^{\left(3 l^{2}+1\right)(j-1) h_{n} /\left(1-\left(3 l^{2}+1\right) h_{n}\right)},
\end{aligned}
$$

for all $j=1, \ldots, n$, provided that $h_{n}<1 /\left(3 l^{2}+1\right)$. But, since $h_{n}$ is intended to tend towards zero, we can, without loss of generality, consider that $h_{n} \leqslant 1 / 2\left(3 l^{2}+1\right)$ with $h_{n} \leqslant T$ of course. In this case, inequality (4.31) implies, for all $j=1, \ldots, n$, that

$$
\left\|\delta^{2} u_{j}\right\|_{B_{2}^{1}}^{2}+\left\|\delta u_{j}\right\|^{2} \leqslant 2\left\{\left\|U_{1}\right\|^{2}+2\left[c_{1}+\left\|\frac{d^{2} U_{0}}{d x^{2}}\right\|_{B_{2}^{1}}^{2}\right]+3 l^{2} T\right\} e^{2\left(3 l^{2}+1\right) T}
$$

if $1 / 2\left(3 l^{2}+1\right) \leqslant T$, and

$$
\left\|\delta^{2} u_{j}\right\|_{B_{2}^{1}}^{2}+\left\|\delta u_{j}\right\|^{2} \leqslant \frac{\left\|U_{1}\right\|^{2}+2\left[c_{1}+\left\|d^{2} U_{0} / d x^{2}\right\|_{B_{2}^{1}}^{2}\right]+3 l^{2} T}{1-\left(3 l^{2}+1\right) T} e^{\left(3 l^{2}+1\right) T / 2\left(1-\left(3 l^{2}+1\right) T\right)}
$$


otherwise. Estimates (4.2) and (4.3) then follow with

$$
c:=c_{2}:=\left\{\begin{array}{l}
\sqrt{2\left\{\left\|U_{1}\right\|^{2}+2\left[c_{1}+\left\|\frac{d^{2} U_{0}}{d x^{2}}\right\|_{B_{2}^{1}}^{2}\right]+3 l^{2} T\right\}} e^{\left(3 l^{2}+1\right) T}, \quad \text { if } T \geqslant \frac{1}{2\left(3 l^{2}+1\right)}, \\
\sqrt{\frac{\left\|U_{1}\right\|^{2}+2\left[C_{1}+\left\|d^{2} U_{0} / d x^{2}\right\|_{B_{2}^{1}}^{2}\right]+3 l^{2} T}{1-\left(3 l^{2}+1\right) T}} e^{\left(3 l^{2}+1\right) T / 2\left(1-\left(3 l^{2}+1\right) T\right)}, \\
\text { if } T<\frac{1}{2\left(3 l^{2}+1\right)},
\end{array}\right.
$$

for all $n \geqslant n_{0}$, where $n_{0}$ is any positive integer such that $T / n_{0} \leqslant 1 / 2\left(3 l^{2}+1\right)$, that is, $n_{0} \geqslant$ $2 T\left(3 l^{2}+1\right)$.

Finally, from the identity

$$
u_{j}=U_{0}+h_{n} \sum_{i=1}^{j} \delta u_{i}, \quad \forall j=1, \ldots, n,
$$

we deduce in light of what precedes that

$$
\left\|u_{j}\right\| \leqslant\left\|U_{0}\right\|+h_{n} \sum_{i=1}^{j}\left\|\delta u_{i}\right\| \leqslant\left\|U_{0}\right\|+j h_{n} c_{2}
$$

hence

$$
\left\|u_{j}\right\| \leqslant\left\|U_{0}\right\|+T c_{2}:=c, \quad \forall j=1, \ldots, n,
$$

which finishes the proof.

As a consequence of Lemma 4.1, we have the following corollary.

Corollary 4.2. There exist $c>0$ such that the estimates

$$
\begin{gathered}
\left\|u^{n}(t)\right\| \leqslant c, \quad\left\|\bar{u}^{n}(t)\right\| \leqslant c, \quad\left\|\frac{d u^{n}}{d t}(t)\right\| \leqslant c, \\
\left\|\bar{u}^{n}(t)-u^{n}(t)\right\| \leqslant c h_{n}, \quad\left\|u^{n}(t)-\bar{u}^{n}\left(t-h_{n}\right)\right\| \leqslant c h_{n}, \\
\left\|\delta u^{n}(t)\right\| \leqslant c, \quad\left\|\overline{s u}^{n}(t)\right\| \leqslant c, \quad\left\|\frac{d}{d t} \delta u^{n}(t)\right\|_{B_{2}^{1}} \leqslant c, \\
\left\|\overline{\delta u}^{n}(t)-\delta u^{n}(t)\right\|_{B_{2}^{1}} \leqslant c h_{n}, \quad\left\|\delta u^{n}(t)-\overline{\delta u}^{n}\left(t-h_{n}\right)\right\|_{B_{2}^{1}} \leqslant c h_{n}, \\
\left\|\delta u^{n}-\frac{d u^{n}}{d t}\right\|_{L^{2}\left(I, B_{2}^{1}\right)} \leqslant c h_{n}
\end{gathered}
$$

hold for all $t \in I$ and $n \geqslant n_{0}$. 
224 On a quasilinear wave equation with integral conditions

Proof. Obviously, estimates $(4.38)_{1}$ and $(4.38)_{2}$ are a direct consequence of (4.1), while estimates $(4.40)_{1}$ and $(4.40)_{2}$ follow immediately from (4.2). On the other hand, since

$$
\begin{aligned}
\frac{d u^{n}}{d t}(t) & = \begin{cases}\delta u_{j}, & \forall t \in\left(t_{j-1}, t_{j}\right], 1 \leqslant j \leqslant n, \\
\delta u_{1}, & t=0,\end{cases} \\
\bar{u}^{n}(t)-u^{n}(t) & = \begin{cases}\delta u_{j}\left(t_{j}-t\right), & \forall t \in\left(t_{j-1}, t_{j}\right], 1 \leqslant j \leqslant n, \\
0, & t=0,\end{cases} \\
u^{n}(t)-\bar{u}^{n}\left(t-h_{n}\right) & = \begin{cases}\delta u_{j}\left(t-t_{j-1}\right), & \forall t \in\left(t_{j-1}, t_{j}\right], 1 \leqslant j \leqslant n, \\
0, & t=0,\end{cases}
\end{aligned}
$$

we derive

$$
\begin{gathered}
\left\|\frac{d u^{n}}{d t}(t)\right\| \leqslant \max _{1 \leqslant j \leqslant n}\left\|\delta u_{j}\right\|, \\
\left\|\bar{u}^{n}(t)-u^{n}(t)\right\| \leqslant h_{n} \max _{1 \leqslant j \leqslant n}\left\|\delta u_{j}\right\|, \\
\left\|u^{n}(t)-\bar{u}^{n}\left(t-h_{n}\right)\right\| \leqslant h_{n} \max _{1 \leqslant j \leqslant n}\left\|\delta u_{j}\right\|,
\end{gathered}
$$

from which estimates $(4.38)_{3}$ and (4.39) follow, thanks to (4.2). Similarly, from the identities

$$
\begin{aligned}
& \frac{d}{d t} \delta u^{n}(t)= \begin{cases}\delta^{2} u_{j}, & \forall t \in\left(t_{j-1}, t_{j}\right], 1 \leqslant j \leqslant n \\
\delta^{2} u_{1}, & t=0\end{cases} \\
& \overline{\delta u}^{n}(t)-\delta u^{n}(t)= \begin{cases}\delta^{2} u_{j}\left(t_{j}-t\right), & \forall t \in\left(t_{j-1}, t_{j}\right], 1 \leqslant j \leqslant n, \\
0, & t=0,\end{cases} \\
& \delta u^{n}(t)-\overline{\delta u}^{n}\left(t-h_{n}\right)= \begin{cases}\delta^{2} u_{j}\left(t-t_{j-1}\right), & \forall t \in\left(t_{j-1}, t_{j}\right], 1 \leqslant j \leqslant n, \\
0, & t=0,\end{cases} \\
& \delta u^{n}(t)-\frac{d u^{n}}{d t}(t)=\delta^{2} u_{j}\left(t-t_{j}\right), \quad \forall t \in\left(t_{j-1}, t_{j}\right], 1 \leqslant j \leqslant n,
\end{aligned}
$$

we deduce the remaining estimates $(4.40)_{3},(4.41)$, and (4.42) in view of (4.3).

\section{Convergence and existence result}

We define, for all $n \geqslant n_{0}$, the abstract function $\bar{f}^{(n)}: I \times V \times V \rightarrow L^{2}(0,1)$ by

$$
\bar{f}^{n}(t, w, p)=f\left(t_{j}, w, p\right), \quad \forall t \in\left(t_{j-1}, t_{j}\right], j=1, \ldots, n .
$$

Then the variational equations $(4.9)_{j}$ may be written anew as

$$
\left(\frac{d}{d t} \delta u^{n}(t), \phi\right)_{B_{2}^{1}}+\left(\bar{u}^{n}(t), \phi\right)=\left(\bar{f}^{n}\left(t, \bar{u}^{n}\left(t-h_{n}\right), \overline{\delta u}^{n}\left(t-h_{n}\right)\right), \phi\right)_{B_{2}^{1}},
$$

for all $\phi \in V$ and all $t \in(0, T]$. 
Before we pass to the limit $n \rightarrow \infty$ in the approximation scheme $(5.2)^{n}$, we must establish some convergence assertions.

Theorem 5.1. There exists a function $u \in C^{0,1}(I, V)$ with $d u / d t \in L^{\infty}(I, V) \cap C^{0,1}\left(I, B_{2}^{1}(0,1)\right)$ and $d^{2} u / d t^{2} \in L^{\infty}\left(I, B_{2}^{1}(0,1)\right)$ such that

(i) $u^{n} \rightarrow u$ in $C(I, V)$;

(ii) $\bar{u}^{n}(t) \rightarrow u(t)$ in $V$ for all $t \in I$;

(iii) $\delta u^{n} \rightarrow d u / d t$ in $C\left(I, B_{2}^{1}(0,1)\right)$;

(iv) $\overline{\delta u}^{n} \rightarrow d u / d t$ in $V$ for all $t \in I$;

(v) $d u^{n} / d t \rightarrow d u / d t$ in $L^{2}(I, V)$;

(vi) $(d / d t) \delta u^{n} \rightarrow d^{2} u / d t^{2}$ in $L^{2}\left(I, B_{2}^{1}(0,1)\right)$.

Moreover, the error estimate

$$
\left\|u^{n}-u\right\|_{C(I, V)}+\left\|\delta u^{n}-\frac{d u}{d t}\right\|_{C\left(I, B_{2}^{1}\right)} \leqslant c h_{n}^{1 / 2}
$$

takes place for all $n \geqslant n_{0}$.

Proof. The key point to the proof is to show that $\left\{u^{n}\right\}_{n}$ and $\left\{\delta u^{n}\right\}_{n}$ are Cauchy sequences in the Banach spaces $C(I, V)$ and $C\left(I, B_{2}^{1}(0,1)\right)$, respectively. For this, we consider the Rothe functions (3.18) $u^{n}$ and $u^{m}$ corresponding to the step lengths $h_{n}=T / n$ and $h_{m}=T / m$, respectively, with $m>n \geqslant n_{0}$. Putting $\phi=\overline{\delta u}^{n, m}(t):=\overline{\delta u}^{n}(t)-\overline{\delta u}^{m}(t)$ in the difference $(5.2)^{n}-(5.2)^{m}$, we get, for all $t \in(0, T]$,

$$
\left(\frac{d}{d t}\left(\delta u^{n}(t)-\delta u^{m}(t)\right), \overline{\delta u}^{n, m}(t)\right)_{B_{2}^{1}}+\left(\bar{u}^{n}(t)-\bar{u}^{m}(t), \overline{\delta u}^{n, m}(t)\right)=\left(\bar{f}^{n}-\bar{f}^{m}, \overline{\delta u}^{n, m}(t)\right)_{B_{2}^{1}},
$$

where the abbreviation

$$
\bar{f}^{n}:=\bar{f}^{n}\left(t, \bar{u}^{n}\left(t-h_{n}\right), \overline{\delta u}^{n}\left(t-h_{n}\right)\right)
$$

has been used. Observing that

$$
\begin{gathered}
\bar{u}^{n}-\bar{u}^{m}=\left(\bar{u}^{n}-u^{n}\right)+\left(u^{n}-u^{m}\right)+\left(u^{m}-\bar{u}^{m}\right), \\
\frac{d u^{n}}{d t}(t)=\overline{\delta u}^{n}(t), \quad \forall t \in(0, T],
\end{gathered}
$$

we can write

$$
\begin{aligned}
\left(\bar{u}^{n}(t)-\bar{u}^{m}(t), \overline{\delta u}^{n, m}(t)\right)= & \left(\left(\bar{u}^{n}(t)-u^{n}(t)\right)+\left(u^{m}(t)-\bar{u}^{m}(t)\right), \overline{\delta u}^{n, m}(t)\right) \\
& +\left(u^{n}(t)-u^{m}(t), \frac{d}{d t}\left(u^{n}(t)-u^{m}(t)\right)\right) \\
= & \left(\left(\bar{u}^{n}(t)-u^{n}(t)\right)+\left(u^{m}(t)-\bar{u}^{m}(t)\right), \overline{\delta u}^{n, m}(t)\right) \\
& +\frac{1}{2} \frac{d}{d t}\left\|u^{n}(t)-u^{m}(t)\right\|^{2}, \quad \text { for a.e. } t \in I .
\end{aligned}
$$


Analogously, we have

$$
\begin{aligned}
\left(\frac{d}{d t}(\right. & \left.\left.\delta u^{n}(t)-\delta u^{m}(t)\right), \overline{\delta u}^{n, m}(t)\right)_{B_{2}^{1}} \\
= & \left(\frac{d}{d t}\left(\delta u^{n}(t)-\delta u^{m}(t)\right),\left(\overline{\delta u}^{n}(t)-\delta u^{n}(t)\right)+\left(\delta u^{m}(t)-\overline{\delta u}^{m}(t)\right)\right)_{B_{2}^{1}} \\
& +\left(\frac{d}{d t}\left(\delta u^{n}(t)-\delta u^{m}(t)\right), \delta u^{n}(t)-\delta u^{m}(t)\right)_{B_{2}^{1}} \\
= & \left(\frac{d}{d t}\left(\delta u^{n}(t)-\delta u^{m}(t)\right),\left(\overline{\delta u}^{n}(t)-\delta u^{n}(t)\right)+\left(\delta u^{m}(t)-\overline{\delta u}^{m}(t)\right)\right)_{B_{2}^{1}} \\
& +\frac{1}{2} \frac{d}{d t}\left\|\delta u^{n}(t)-\delta u^{m}(t)\right\|_{B_{2}^{1}}^{2}, \quad \text { for a.e. } t \in I .
\end{aligned}
$$

Substituting (5.7) and (5.8) in (5.4) and rearranging, we obtain

$$
\begin{aligned}
\frac{1}{2} \frac{d}{d t}\left\|u^{n}(t)-u^{m}(t)\right\|^{2}+\frac{1}{2} \frac{d}{d t}\left\|\delta u^{n}(t)-\delta u^{m}(t)\right\|_{B_{2}^{1}}^{2} \\
=\left(\frac{d}{d t}\left(\delta u^{n}(t)-\delta u^{m}(t)\right),\left(\delta u^{n}(t)-\overline{\delta u}^{n}(t)\right)+\left(\overline{\delta u}^{m}(t)-\delta u^{m}(t)\right)\right)_{B_{2}^{1}} \\
\quad+\left(\left(u^{n}(t)-\bar{u}^{n}(t)\right)+\left(\bar{u}^{m}(t)-u^{m}(t)\right), \overline{\delta u}^{n, m}(t)\right)+\left(\bar{f}^{n}-\bar{f}^{m}, \overline{\delta u}^{n, m}(t)\right)_{B_{2}^{1}} .
\end{aligned}
$$

Estimating the first two terms in the right-hand side, we write

$$
\begin{aligned}
\left(\frac{d}{d t}\left(\delta u^{n}(t)-\delta u^{m}(t)\right),\left(\delta u^{n}(t)-\overline{\delta u}^{n}(t)\right)+\left(\overline{\delta u}^{m}(t)-\delta u^{m}(t)\right)\right)_{B_{2}^{1}} \\
\leqslant\left(\left\|\frac{d}{d t} \delta u^{n}(t)\right\|_{B_{2}^{1}}+\left\|\frac{d}{d t} \delta u^{m}(t)\right\|_{B_{2}^{1}}\right) \\
\quad \times\left(\left\|\delta u^{n}(t)-\overline{\delta u}^{n}(t)\right\|_{B_{2}^{1}}+\left\|\overline{\delta u}{ }^{m}(t)-\delta u^{m}(t)\right\|_{B_{2}^{1}}\right) \\
\leqslant c\left(h_{n}+h_{m}\right)
\end{aligned}
$$

in view of $(4.40)_{3}$ and $(4.41)_{1}$. Similarly,

$$
\begin{aligned}
& \left(\left(u^{n}(t)-\bar{u}^{n}(t)\right)+\left(\bar{u}^{m}(t)-u^{m}(t)\right), \overline{\delta u}^{n, m}(t)\right) \\
& \quad \leqslant\left(\left\|u^{n}(t)-\bar{u}^{n}(t)\right\|+\left\|\bar{u}^{m}(t)-u^{m}(t)\right\|\right)\left(\left\|\overline{\delta u}^{n}(t)\right\|+\left\|\overline{\delta u}^{m}(t)\right\|\right) \\
& \quad \leqslant c\left(h_{n}+h_{m}\right)
\end{aligned}
$$

in view of $(4.39)_{1}$ and $(4.40)_{2}$. It remains to dominate the last term in the right-hand side in (5.9). For any $t$ fixed in $(0, T]$, there exist two integers $k$ and $i$ corresponding to the subdivision of $I$ into $n$ and $m$ subintervals, respectively, such that $t \in\left(t_{k-1}, t_{k}\right] \cap\left(t_{i-1}, t_{i}\right]$. 
Consequently, owing to assumption $\left(\mathrm{H}_{1}\right)$, it follows that

$$
\begin{aligned}
\left\|\bar{f}^{n}-\bar{f}^{m}\right\|_{B_{2}^{1}} & \\
= & \left\|\bar{f}^{n}\left(t_{k}, \bar{u}^{n}\left(t-h_{n}\right), \overline{\delta u}^{n}\left(t-h_{n}\right)\right)-\bar{f}^{m}\left(t_{i}, \bar{u}^{m}\left(t-h_{m}\right), \overline{\delta u}^{m}\left(t-h_{m}\right)\right)\right\|_{B_{2}^{1}} \\
\leqslant & l\left(\left|t_{k}-t_{i}\right|+\left\|\bar{u}^{n}\left(t-h_{n}\right)-\bar{u}^{m}\left(t-h_{m}\right)\right\|_{B_{2}^{1}}+\left\|\overline{\delta u}^{n}\left(t-h_{n}\right)-\overline{\delta u}^{m}\left(t-h_{m}\right)\right\|_{B_{2}^{1}}\right) \\
\leqslant & l\left(h_{n}+h_{m}+\left\|\bar{u}^{n}\left(t-h_{n}\right)-u^{n}(t)\right\|_{B_{2}^{1}}+\left\|u^{n}(t)-u^{m}(t)\right\|_{B_{2}^{1}}+\left\|u^{m}(t)-\bar{u}^{m}\left(t-h_{m}\right)\right\|_{B_{2}^{1}}\right. \\
& \left.\quad+\left\|\overline{\delta u}^{n}\left(t-h_{n}\right)-\delta u^{n}(t)\right\|_{B_{2}^{1}}+\left\|\delta u^{n}(t)-\delta u^{m}(t)\right\|_{B_{2}^{1}}+\left\|\delta u^{m}(t)-\overline{\delta u}^{m}\left(t-h_{m}\right)\right\|_{B_{2}^{1}}\right),
\end{aligned}
$$

then, according to $(4.39)_{2}$ and $(4.41)_{2}$, we have

$$
\left\|\bar{f}^{n}-\bar{f}^{m}\right\|_{B_{2}^{1}} \leqslant c\left(h_{n}+h_{m}\right)+l\left(\left\|u^{n}(t)-u^{m}(t)\right\|_{B_{2}^{1}}+\left\|\delta u^{n}(t)-\delta u^{m}(t)\right\|_{B_{2}^{1}}\right) .
$$

On the other hand, due to $(4.41)_{1}$, we estimate

$$
\begin{aligned}
& \left\|\overline{\delta u}^{n, m}(t)\right\|_{B_{2}^{1}} \\
& \quad \leqslant\left\|\overline{\delta u}^{n}(t)-\delta u^{n}(t)\right\|_{B_{2}^{1}}+\left\|\delta u^{n}(t)-\delta u^{m}(t)\right\|_{B_{2}^{1}}+\left\|\delta u^{m}(t)-\overline{\delta u}^{m}(t)\right\|_{B_{2}^{1}} \\
& \quad \leqslant c\left(h_{n}+h_{m}\right)+\left\|\delta u^{n}(t)-\delta u^{m}(t)\right\|_{B_{2}^{1}} .
\end{aligned}
$$

From (5.13) and (5.14), we conclude, thanks to $(4.40)_{1}$ and $(4.38)_{1}$, that

$$
\begin{aligned}
&\left(\bar{f}^{n}-\bar{f}^{m}, \overline{\delta u}^{n, m}(t)\right)_{B_{2}^{1}} \\
& \leqslant\left\|\bar{f}^{n}-\bar{f}^{m}\right\|_{B_{2}^{1}}\left\|\overline{\delta u}{ }^{n, m}(t)\right\|_{B_{2}^{1}} \\
& \leqslant c\left(h_{n}+h_{m}\right)^{2}+c\left(h_{n}+h_{m}\right)\left\|\delta u^{n}(t)-\delta u^{m}(t)\right\|_{B_{2}^{1}} \\
&+c\left(h_{n}+h_{m}\right)\left(\left\|u^{n}(t)-u^{m}(t)\right\|_{B_{2}^{1}}+\left\|\delta u^{n}(t)-\delta u^{m}(t)\right\|_{B_{2}^{1}}\right) \\
&+l\left\|u^{n}(t)-u^{m}(t)\right\|_{B_{2}^{1}}\left\|\delta u^{n}(t)-\delta u^{m}(t)\right\|_{B_{2}^{1}}+l\left\|\delta u^{n}(t)-\delta u^{m}(t)\right\|_{B_{2}^{1}}^{2} \\
& \leqslant c\left(h_{n}+h_{m}\right)^{2}+c\left(h_{n}+h_{m}\right)\left(\left\|\delta u^{n}(t)\right\|_{B_{2}^{1}}+\left\|\delta u^{m}(t)\right\|_{B_{2}^{1}}\right) \\
&+c\left(h_{n}+h_{m}\right)\left(\left\|u^{n}(t)\right\|_{B_{2}^{1}}+\left\|u^{m}(t)\right\|_{B_{2}^{1}}+\left\|\delta u^{n}(t)\right\|_{B_{2}^{1}}+\left\|\delta u^{m}(t)\right\|_{B_{2}^{1}}\right) \\
&+\frac{l}{2}\left(\left\|u^{n}(t)-u^{m}(t)\right\|_{B_{2}^{1}}^{2}+\left\|\delta u^{n}(t)-\delta u^{m}(t)\right\|_{B_{2}^{1}}^{2}\right)+l\left\|\delta u^{n}(t)-\delta u^{m}(t)\right\|_{B_{2}^{1}}^{2}
\end{aligned}
$$

here, the elementary inequality $a b \leqslant(1 / 2)\left(a^{2}+b^{2}\right)$ has been used. Hence,

$$
\begin{aligned}
& \left(\bar{f}^{n}-\bar{f}^{m}, \overline{\delta u}^{n, m}(t)\right)_{B_{2}^{1}} \\
& \quad \leqslant c\left(h_{n}+h_{m}\right)^{2}+c\left(h_{n}+h_{m}\right)+\frac{l}{2}\left\|u^{n}(t)-u^{m}(t)\right\|_{B_{2}^{1}}^{2}+\frac{3 l}{2}\left\|\delta u^{n}(t)-\delta u^{m}(t)\right\|_{B_{2}^{1}}^{2} .
\end{aligned}
$$


228 On a quasilinear wave equation with integral conditions

Combining (5.9), (5.10), (5.11), and (5.16), we obtain for a.e. $t \in I$,

$$
\begin{aligned}
& \frac{d}{d t}\left\|u^{n}(t)-u^{m}(t)\right\|^{2}+\frac{d}{d t}\left\|\delta u^{n}(t)-\delta u^{m}(t)\right\|_{B_{2}^{1}}^{2} \\
& \quad \leqslant c\left(h_{n}+h_{m}\right)^{2}+c\left(h_{n}+h_{m}\right)+l\left\|u^{n}(t)-u^{m}(t)\right\|^{2}+3 l\left\|\delta u^{n}(t)-\delta u^{m}(t)\right\|_{B_{2}^{1}}^{2} .
\end{aligned}
$$

Integrating over $(0, t)$ with consideration to the fact that

$$
\begin{gathered}
u^{n}(0)=u^{m}(0)=U_{0}, \\
\delta u^{n}(0)=\delta u^{m}(0)=U_{1},
\end{gathered}
$$

we have

$$
\begin{aligned}
\| u^{n}(t)- & u^{m}(t)\left\|^{2}+\right\| \delta u^{n}(t)-\delta u^{m}(t) \|_{B_{2}^{1}}^{2} \\
\leqslant & c\left(h_{n}+h_{m}\right)^{2}+c\left(h_{n}+h_{m}\right)+l \int_{0}^{t}\left\|u^{n}(\tau)-u^{m}(\tau)\right\|^{2} d \tau \\
& +3 l \int_{0}^{t}\left\|\delta u^{n}(\tau)-\delta u^{m}(\tau)\right\|_{B_{2}^{1}}^{2} d \tau, \quad \forall t \in I
\end{aligned}
$$

or, by Gronwall's lemma,

$$
\left\|u^{n}(t)-u^{m}(t)\right\|^{2}+\left\|\delta u^{n}(t)-\delta u^{m}(t)\right\|_{B_{2}^{1}}^{2} \leqslant\left(c\left(h_{n}+h_{m}\right)^{2}+c\left(h_{n}+h_{m}\right)\right) e^{3 l T}, \quad \forall t \in I .
$$

Hence, taking the upper bound with respect to $t \in I$ in the left-hand side of this last inequality, we obtain

$$
\left\|u^{n}-u^{m}\right\|_{C(I, V)}^{2}+\left\|\delta u^{n}-\delta u^{m}\right\|_{C\left(I, B_{2}\right)}^{2} \leqslant\left(c\left(h_{n}+h_{m}\right)^{2}+c\left(h_{n}+h_{m}\right)\right) e^{3 l T}
$$

from which we deduce that both $\left\{u^{n}\right\}_{n}$ and $\left\{\delta u^{n}\right\}_{n}$ are Cauchy sequences in the Banach spaces $C(I, V)$ and $C\left(I, B_{2}^{1}(0,1)\right)$, respectively. Accordingly, there exist two functions $u \in$ $C(I, V)$ and $w \in C\left(I, B_{2}^{1}(0,1)\right)$ such that

$$
\begin{aligned}
u^{n} \longrightarrow u & \text { in } C(I, V), \\
\delta u^{n} \longrightarrow w & \text { in } C\left(I, B_{2}^{1}(0,1)\right) .
\end{aligned}
$$

Now, on the basis of estimations $(4.38)_{2},(4.38)_{3},(4.39)_{1}$ and the convergence result (5.22), [18, Lemma 1.3.15] enables us to state the following assertions:

(i) $u \in C^{0,1}(I, V)$;

(ii) $u$ is strongly differentiable a.e. in $I$ and $d u / d t \in L^{\infty}(I, V)$;

(iii) $\bar{u}^{n}(t) \rightarrow u(t)$ in $V$ for all $t \in I$;

(iv) $d u^{n} / d t \rightarrow d u / d t$ in $L^{2}(I, V)$.

On the other hand, in light of estimations $(4.40)_{2},(4.40)_{3}$, the convergence statement (5.23), and the continuous embedding $V \hookrightarrow B_{2}^{1}(0,1)$, [18, Lemma 1.3.15] is also valid 
for the functions $\delta u^{n}$ and the corresponding step functions $\overline{\delta u}^{n}$, yielding the following statements:

(v) $w \in C^{0,1}\left(I, B_{2}^{1}(0,1)\right)$;

(vi) $w$ is strongly differentiable a.e. in $I$ and $d w / d t \in L^{\infty}\left(I, B_{2}^{1}(0,1)\right)$;

(vii) $\overline{\delta u}^{n}(t) \rightarrow w(t)$ in $V$ for all $t \in I$;

(viii) $(d / d t) \delta u^{n} \rightarrow d w / d t$ in $L^{2}\left(I, B_{2}^{1}(0,1)\right)$.

We show that $w$ coincides with $d u / d t$. For all $v \in L^{2}\left(I, B_{2}^{1}(0,1)\right)$, we have

$$
\begin{aligned}
\left(\delta u^{n}-\frac{d u}{d t}, v\right)_{L^{2}\left(I, B_{2}^{1}\right)} & =\left(\delta u^{n}-\frac{d u^{n}}{d t}, v\right)_{L^{2}\left(I, B_{2}^{1}\right)}+\left(\frac{d u^{n}}{d t}-\frac{d u}{d t}, v\right)_{L^{2}\left(I, B_{2}^{1}\right)} \\
& \leqslant\left\|\delta u^{n}-\frac{d u^{n}}{d t}\right\|_{L^{2}\left(I, B_{2}^{1}\right)}\|v\|_{L^{2}\left(I, B_{2}^{1}\right)}+\left(\frac{d u^{n}}{d t}-\frac{d u}{d t}, v\right)_{L^{2}\left(I, B_{2}^{1}\right)}
\end{aligned}
$$

or, due to (4.42) and the convergence property (iv) stated above,

$$
\left(\delta u^{n}-\frac{d u}{d t}, v\right)_{L^{2}\left(I, B_{2}^{1}\right)} \leqslant c h_{n}\|v\|_{L^{2}\left(I, B_{2}^{1}\right)}+\left(\frac{d u^{n}}{d t}-\frac{d u}{d t}, v\right)_{L^{2}\left(I, B_{2}^{1}\right)} \longrightarrow 0
$$

as $n \rightarrow \infty$; hence

$$
\delta u^{n} \rightarrow \frac{d u}{d t} \quad \text { in } L^{2}\left(I, B_{2}^{1}(0,1)\right)
$$

which, together with (5.23), yields $w=d u / d t$ and consequently $d w / d t=d^{2} u / d t^{2}$. Finally, letting $m \rightarrow \infty$ in (5.21), taking into account that $h_{n} \leqslant h_{n_{0}} \leqslant 1 / 2$, we obtain the desired error estimate. So, the proof is complete.

Now, we are ready to state an existence result.

Theorem 5.2. The limit function $u$ from Theorem 5.1 is the unique weak solution to problem (1.5)-(1.8) in the sense of Definition 2.2.

Proof

Existence. We have to show that the limit function $u$ satisfies all the conditions (i), (ii), (iii), (iv) of Definition 2.2. Obviously, in light of the properties of the function $u$ listed in Theorem 5.1, the first two conditions of Definition 2.2 are already seen. On the other hand, since $u^{n} \rightarrow u$ in $C(I, V)$ and $\delta u^{n} \rightarrow d u / d t$ in $C\left(I, B_{2}^{1}(0,1)\right)$ as $n \rightarrow \infty$ and, by construction, $u^{n}(0)=U_{0}$ and $\delta u^{n}(0)=U_{1}$, it follows that $u(0)=U_{0}$ and $(d u / d t)(0)=U_{1}$ hold in $V$ and $B_{2}^{1}(0,1)$, respectively, so the initial conditions (1.6) are also fulfilled, that is, Definition 2.2(iii) takes place. It remains to see that the integral identity $(2.13)$ is obeyed by $u$. For this, we consider the following relation:

$$
\begin{aligned}
& \left(\delta u^{n}(t)-U_{1}, \phi\right)_{B_{2}^{1}}+\int_{0}^{t}\left(\bar{u}^{n}(\tau), \phi\right) d \tau \\
& \quad=\int_{0}^{t}\left(\bar{f}^{n}\left(\tau, \bar{u}^{n}\left(\tau-h_{n}\right), \overline{\delta u}^{n}\left(\tau-h_{n}\right)\right), \phi\right)_{B_{2}^{1}} d \tau, \quad \forall \phi \in V, \forall t \in I,
\end{aligned}
$$


which results from $(5.2)^{n}$ by integration between 0 and $t \in I$, noting that $\delta u^{n}(0)=U_{1}$. First, by virtue of Theorem 5.1(iii), we have

$$
\left(\delta u^{n}(t)-U_{1}, \phi\right)_{B_{2}^{1}} \underset{n \rightarrow \infty}{\longrightarrow}\left(\frac{d u}{d t}(t)-U_{1}, \phi\right)_{B_{2}^{1}}, \quad \forall \phi \in V, \forall t \in I .
$$

Next, according to estimate $(4.38)_{2}$, the expression $\left|\left(\bar{u}^{n}(\tau), \phi\right)\right|$ is uniformly bounded with respect to both $n$ and $\tau$, so the Lebesgue theorem of dominated convergence may be applied to the convergence statement (ii) from Theorem 5.1, yielding

$$
\int_{0}^{t}\left(\bar{u}^{n}(\tau), \phi\right) d \tau \underset{n \rightarrow \infty}{\longrightarrow} \int_{0}^{t}(u(\tau), \phi) d \tau, \quad \forall \phi \in V, \forall t \in I .
$$

To investigate the behavior of the right-hand side of (5.27) as $n \rightarrow \infty$, we first observe that for all $\tau \in\left(t_{j-1}, t_{j}\right], 1 \leqslant j \leqslant n$, we have

$$
\begin{aligned}
& \left\|\bar{f}^{n}\left(\tau, \bar{u}^{n}\left(\tau-h_{n}\right), \overline{\delta u}^{n}\left(\tau-h_{n}\right)\right)-f\left(\tau, u(\tau), \frac{d u}{d t}(\tau)\right)\right\|_{B_{2}^{1}} \\
& \quad=\left\|f\left(t_{j}, \bar{u}^{n}\left(\tau-h_{n}\right), \overline{\delta u}^{n}\left(\tau-h_{n}\right)\right)-f\left(\tau, u(\tau), \frac{d u}{d t}(\tau)\right)\right\|_{B_{2}^{1}} \\
& \quad \leqslant l\left[\left|t_{j}-\tau\right|+\left\|\bar{u}^{n}\left(\tau-h_{n}\right)-u(\tau)\right\|_{B_{2}^{1}}+\left\|\overline{\delta u}^{n}\left(\tau-h_{n}\right)-\frac{d u}{d t}(\tau)\right\|_{B_{2}^{1}}\right],
\end{aligned}
$$

owing to assumption $\left(\mathrm{H}_{1}\right)$. However, from estimates $(4.39)_{2}$ and (5.3), we derive

$$
\begin{aligned}
\left\|\bar{u}^{n}\left(\tau-h_{n}\right)-u(\tau)\right\|_{B_{2}^{1}} & \leqslant\left\|\bar{u}^{n}\left(\tau-h_{n}\right)-u^{n}(\tau)\right\|_{B_{2}^{1}}+\left\|u^{n}(\tau)-u(\tau)\right\|_{B_{2}^{1}} \\
& \leqslant c\left(h_{n}+h_{n}^{1 / 2}\right), \quad \forall \tau \in I ;
\end{aligned}
$$

similarly, from estimates $(4.41)_{2}$ and (5.3), we get

$$
\begin{aligned}
\left\|\overline{\delta u}^{n}\left(\tau-h_{n}\right)-\frac{d u}{d t}(\tau)\right\|_{B_{2}^{1}} & \leqslant\left\|\overline{\delta u}^{n}\left(\tau-h_{n}\right)-\delta u^{n}(\tau)\right\|_{B_{2}^{1}}+\left\|\delta u^{n}(\tau)-\frac{d u}{d t}(\tau)\right\|_{B_{2}^{1}} \\
& \leqslant c\left(h_{n}+h_{n}^{1 / 2}\right), \quad \forall \tau \in I .
\end{aligned}
$$

Therefore, for all $\tau \in(0, T]$, it holds that

$$
\left\|\bar{f}^{n}\left(\tau, \bar{u}^{n}\left(\tau-h_{n}\right), \overline{\delta u}^{n}\left(\tau-h_{n}\right)\right)-f\left(\tau, u(\tau), \frac{d u}{d t}(\tau)\right)\right\|_{B_{2}^{1}} \leqslant c\left(h_{n}+h_{n}^{1 / 2}\right),
$$


from which we deduce that

$$
\bar{f}^{n}\left(\tau, \bar{u}^{n}\left(\tau-h_{n}\right), \overline{\delta u}^{n}\left(\tau-h_{n}\right)\right) \underset{n \rightarrow \infty}{\longrightarrow} f\left(\tau, u(\tau), \frac{d u}{d t}(\tau)\right) \quad \text { in } B_{2}^{1}(0,1), \forall \tau \in(0, T] .
$$

But, inasmuch as

$$
\bar{f}^{n}\left(\tau, \bar{u}^{n}\left(\tau-h_{n}\right), \overline{\delta u}^{n}\left(\tau-h_{n}\right)\right)=f\left(t_{j}, u_{j-1}, \delta u_{j-1}\right), \quad \forall \tau \in\left(t_{j-1}, t_{j}\right], 1 \leqslant j \leqslant n,
$$

it follows that

$$
\begin{aligned}
& \left\|\bar{f}^{n}\left(\tau, \bar{u}^{n}\left(\tau-h_{n}\right), \overline{\delta u}^{n}\left(\tau-h_{n}\right)\right)\right\|_{B_{2}^{1}} \\
& \quad \leqslant \max _{1 \leqslant j \leqslant n}\left\|f\left(t_{j}, u_{j-1}, \delta u_{j-1}\right)\right\|_{B_{2}^{1}} \\
& \quad \leqslant \max _{1 \leqslant j \leqslant n}\left\|f\left(t_{j}, u_{j-1}, \delta u_{j-1}\right)-f\left(t_{j}, 0,0\right)\right\|_{B_{2}^{1}}+\max _{1 \leqslant j \leqslant n}\left\|f\left(t_{j}, 0,0\right)\right\|_{B_{2}^{1}} \\
& \quad \leqslant l \max _{1 \leqslant j \leqslant n}\left(\left\|u_{j-1}\right\|_{B_{2}^{1}}+\left\|\delta u_{j-1}\right\|_{B_{2}^{1}}\right)+c_{3}, \quad \forall \tau \in(0, T],
\end{aligned}
$$

where $c_{3}:=\max _{1 \leqslant j \leqslant n}\left\|f\left(t_{j}, 0,0\right)\right\|_{B_{2}^{1}}<\infty$. Accordingly,

$$
\left\|\bar{f}^{n}\left(\tau, \bar{u}^{n}\left(\tau-h_{n}\right), \overline{\delta u}^{n}\left(\tau-h_{n}\right)\right)\right\|_{B_{2}^{1}} \leqslant c, \quad \forall \tau \in(0, T],
$$

in view of estimates (4.1) and (4.2). This shows that

$$
\left|\left(\bar{f}^{n}\left(\tau, \bar{u}^{n}\left(\tau-h_{n}\right), \overline{\delta u}^{n}\left(\tau-h_{n}\right)\right), \phi\right)_{B_{2}^{1}}\right|
$$

is uniformly bounded with respect to both $n$ and $\tau$; hence, applying the Lebesgue theorem to the convergence statement (5.34), we get

$$
\int_{0}^{t}\left(\bar{f}^{n}\left(\tau, \bar{u}^{n}\left(\tau-h_{n}\right), \overline{\delta u}^{n}\left(\tau-h_{n}\right)\right), \phi\right)_{B_{2}^{1}} d \tau \underset{n \rightarrow \infty}{\longrightarrow} \int_{0}^{t}\left(f\left(\tau, u(\tau), \frac{d u}{d t}(\tau)\right), \phi\right)_{B_{2}^{1}} d \tau,
$$

for all $\phi \in V$ and all $t \in I$. Finally, performing a limit process $n \rightarrow \infty$ in (5.27), taking into account (5.28), (5.29), and (5.39), we find out that

$$
\begin{aligned}
&\left(\frac{d u}{d t}(\right.\left.t)-U_{1}, \phi\right)_{B_{2}^{1}}+\int_{0}^{t}(u(\tau), \phi) d \tau \\
& \quad=\int_{0}^{t}\left(f\left(\tau, u(\tau), \frac{d u}{d t}(\tau)\right), \phi\right)_{B_{2}^{1}} d \tau, \quad \forall \phi \in V, \forall t \in I
\end{aligned}
$$

But the function $d u / d t: I \rightarrow B_{2}^{1}(0,1)$ is strongly differentiable for a.e. $t \in I$, hence, differentiating the just obtained equality with respect to $t$, we get the desired identity (2.13). Thus, $u$ weakly solves problem (1.5)-(1.8). 
Uniqueness. Let $\hat{u}$ and $\tilde{u}$ be two weak solutions of (1.5)-(1.8). From (2.13), for $u=\hat{u}-\tilde{u}$ and $\phi=(d u / d t)(t)$, we obtain

$$
\begin{aligned}
& \left(\frac{d^{2} u}{d t^{2}}(t), \frac{d u}{d t}(t)\right)_{B_{2}^{1}}+\left(u(t), \frac{d u}{d t}(t)\right) \\
& \quad=\left(f\left(t, \hat{u}(t), \frac{d \hat{u}}{d t}(t)\right)-f\left(t, \tilde{u}(t), \frac{d \tilde{u}}{d t}(t)\right), \frac{d u}{d t}(t)\right)_{B_{2}^{1}}, \quad \text { a.e. } t \in I .
\end{aligned}
$$

So, with consideration to the fact that $(d u / d t)(0)=u(0)=0$, integration from 0 to $t$ yields, in a standard way,

$$
\begin{aligned}
& \frac{1}{2}\left\|\frac{d u}{d t}(t)\right\|_{B_{2}^{1}}^{2}+\frac{1}{2}\|u(t)\|^{2} \\
& \quad \leqslant \int_{0}^{t}\left\|f\left(\tau, \hat{u}(\tau), \frac{d \hat{u}}{d t}(\tau)\right)-f\left(\tau, \tilde{u}(\tau), \frac{d \tilde{u}}{d t}(\tau)\right)\right\|_{B_{2}^{1}}\left\|\frac{d u}{d t}(\tau)\right\|_{B_{2}^{1}} d \tau \\
& \quad \leqslant l \int_{0}^{t}\left[\|u(\tau)\|_{B_{2}^{1}}+\left\|\frac{d u}{d t}(\tau)\right\|_{B_{2}^{1}}\right]\left\|\frac{d u}{d t}(\tau)\right\|_{B_{2}^{1}} d \tau, \\
& \quad \leqslant l \int_{0}^{t}\left[\|u(\tau)\|_{B_{2}^{1}}+\left\|\frac{d u}{d t}(\tau)\right\|_{B_{2}^{1}}\right]^{2} d \tau, \quad \forall t \in I,
\end{aligned}
$$

due to assumption $\left(\mathrm{H}_{1}\right)$, whence

$$
\|u(t)\|^{2}+\left\|\frac{d u}{d t}(t)\right\|_{B_{2}^{1}}^{2} \leqslant 4 l \int_{0}^{t}\left[\|u(\tau)\|^{2}+\left\|\frac{d u}{d t}(\tau)\right\|_{B_{2}^{1}}^{2}\right] d \tau, \quad \forall t \in I,
$$

implying, by Gronwall's lemma, that $u(t)=0, \forall t \in I$, that is, $\hat{u}=\tilde{u}$, which achieves the proof.

We terminate the paper by a result of continuous dependence of the solution $u$ upon data. Concretely, we have the following theorem.

Theorem 5.3. Let $u$ and $u^{*}$ be the weak solutions of problem (1.5)-(1.8), corresponding to $\left(U_{0}, U_{1}, f\right)$ and $\left(U_{0}^{*}, U_{1}^{*}, f^{*}\right)$, respectively. Assume that $\left(U_{0}, U_{1}, f\right)$ and $\left(U_{0}^{*}, U_{1}^{*}, f^{*}\right)$ satisfy assumptions $\left(H_{1}\right),\left(H_{2}\right)$, and $\left(H_{3}\right)$, then the inequality

$$
\begin{aligned}
\| u(t)- & u^{*}(t)\left\|^{2}+\right\| \frac{d u}{d t}(t)-\frac{d u^{*}}{d t}(t) \|_{B_{2}^{1}}^{2} \\
\leqslant & \left(\left\|U_{0}-U_{0}^{*}\right\|^{2}+\left\|U_{1}-U_{1}^{*}\right\|_{B_{2}^{1}}^{2}\right. \\
& \left.\quad+\int_{0}^{t}\left\|f\left(\tau, u(\tau), \frac{d u}{d t}(\tau)\right)-f^{*}\left(\tau, u^{*}(\tau), \frac{d u^{*}}{d t}(\tau)\right)\right\|_{B_{2}^{1}}^{2} d \tau\right) e^{t}
\end{aligned}
$$

takes place for all $t \in I$. 
Proof. Subtracting (2.13) for $u$ and $u^{*}$ and putting $\phi=(d u / d t)(t)-\left(d u^{*} / d t\right)(t)$ in the resulting relation, we get

$$
\begin{aligned}
& \left(\frac{d^{2}}{d t^{2}}\left(u(t)-u^{*}(t)\right), \frac{d}{d t}\left(u(t)-u^{*}(t)\right)\right)_{B_{2}^{1}}+\left(u(t)-u^{*}(t), \frac{d}{d t}\left(u(t)-u^{*}(t)\right)\right) \\
& \quad=\left(f\left(t, u(t), \frac{d u}{d t}(t)\right)-f^{*}\left(t, u^{*}(t), \frac{d u^{*}}{d t}(t)\right), \frac{d}{d t}\left(u(t)-u^{*}(t)\right)\right)_{B_{2}^{1}}, \quad \text { a.e. } t \in I,
\end{aligned}
$$

whence

$$
\begin{aligned}
& \frac{1}{2} \frac{d}{d t}\left\|\frac{d}{d t}\left(u(t)-u^{*}(t)\right)\right\|_{B_{2}^{1}}^{2}+\frac{1}{2} \frac{d}{d t}\left\|u(t)-u^{*}(t)\right\|^{2} \\
& \quad \leqslant\left\|f\left(t, u(t), \frac{d u}{d t}(t)\right)-f^{*}\left(t, u^{*}(t), \frac{d u^{*}}{d t}(t)\right)\right\|_{B_{2}^{1}}\left\|\frac{d}{d t}\left(u(t)-u^{*}(t)\right)\right\|_{B_{2}^{1}},
\end{aligned}
$$

Then, integrating over $(0, t)$, we have

$$
\begin{aligned}
&\left\|\frac{d}{d t}\left(u(t)-u^{*}(t)\right)\right\|_{B_{2}^{1}}^{2}+\left\|u(t)-u^{*}(t)\right\|^{2} \\
& \leqslant\left\|U_{0}-U_{0}^{*}\right\|^{2}+\left\|U_{1}-U_{1}^{*}\right\|_{B_{2}^{1}}^{2} \\
&+2 \int_{0}^{t}\left\|f\left(\tau, u(\tau), \frac{d u}{d t}(\tau)\right)-f^{*}\left(\tau, u^{*}(\tau), \frac{d u^{*}}{d t}(\tau)\right)\right\|_{B_{2}^{1}}\left\|\frac{d}{d t}\left(u(\tau)-u^{*}(\tau)\right)\right\|_{B_{2}^{1}} d \tau \\
& \leqslant\left\|U_{0}-U_{0}^{*}\right\|^{2}+\left\|U_{1}-U_{1}^{*}\right\|_{B_{2}^{1}}^{2} \\
&+\int_{0}^{t}\left\|f\left(\tau, u(\tau), \frac{d u}{d t}(\tau)\right)-f^{*}\left(\tau, u^{*}(\tau), \frac{d u^{*}}{d t}(\tau)\right)\right\|_{B_{2}^{1}}^{2} d \tau \\
&+\int_{0}^{t}\left\|\frac{d}{d t}\left(u(\tau)-u^{*}(\tau)\right)\right\|_{B_{2}^{1}}^{2} d \tau, \quad \forall t \in I,
\end{aligned}
$$

consequently,

$$
\begin{aligned}
\| u(t)- & u^{*}(t)\left\|^{2}+\right\| \frac{d}{d t}\left(u(t)-u^{*}(t)\right) \|_{B_{2}^{1}}^{2} \\
\leqslant & \left\|U_{0}-U_{0}^{*}\right\|^{2}+\left\|U_{1}-U_{1}^{*}\right\|_{B_{2}^{1}}^{2} \\
& +\int_{0}^{t}\left\|f\left(\tau, u(\tau), \frac{d u}{d t}(\tau)\right)-f^{*}\left(\tau, u^{*}(\tau), \frac{d u^{*}}{d t}(\tau)\right)\right\|_{B_{2}^{1}}^{2} d \tau \\
& +\int_{0}^{t}\left[\left\|\frac{d}{d t}\left(u(\tau)-u^{*}(\tau)\right)\right\|_{B_{2}^{1}}^{2}+\left\|u(\tau)-u^{*}(\tau)\right\|^{2}\right] d \tau,
\end{aligned}
$$

for all $t \in I$. Finally, applying the Gronwall lemma, we get inequality (5.44) which represents the continuous dependence of the solution on data. 


\section{References}

[1] S. A. Beilin, Existence of solutions for one-dimensional wave equations with nonlocal conditions, Electron. J. Differential Equations 2001 (2001), no. 76, 1-8.

[2] N.-E. Benouar and N. I. Yurchuk, A mixed problem with an integral condition for parabolic equations with a Bessel operator, Differ. Uravn. 27 (1991), no. 12, 2094-2098 (Russian).

[3] A. Bouziani, Mixed problem with integral condition for certain partial differential equations, Ph.D. thesis, Constantine University, Constantine, Algeria, 1996.

[4] Solution forte d'un problème mixte avec une condition non locale pour une classe d'équations hyperboliques [Strong solution of a mixed problem with a nonlocal condition for a class of hyperbolic equations], Acad. Roy. Belg. Bull. Cl. Sci. (6) 8 (1997), no. 1-6, 53-70 (French).

[5] Strong solution to a hyperbolic evolution problem with nonlocal boundary conditions, Maghreb Math. Rev. 9 (2000), no. 1-2, 71-84.

[6] On the solvability of parabolic and hyperbolic problems with a boundary integral condition, Int. J. Math. Math. Sci. 31 (2002), no. 4, 201-213.

[7] - On initial boundary value problem with Dirichlet integral conditions for a hyperbolic equation with the Bessel operator, J. Appl. Math. 2003 (2003), no. 10, 487-502.

[8] A mixed problem with only integral boundary conditions for a hyperbolic equation, Int. J. Math. Math. Sci. 2004 (2004), no. 24, 1279-1291.

[9] A. Bouziani and N.-E. Benouar, Mixed problem with integral conditions for a third order parabolic equation, Kobe J. Math. 15 (1998), no. 1, 47-58.

[10] J. R. Cannon, The solution of the heat equation subject to the specification of energy, Quart. Appl. Math. 21 (1963), 155-160.

[11] J. R. Cannon and J. van der Hoek, The existence of and a continuous dependence result for the solution of the heat equation subject to the specification of energy, Boll. Un. Mat. Ital. Suppl. 1981 (1981), no. 1, 253-282.

[12] Diffusion subject to the specification of mass, J. Math. Anal. Appl. 115 (1986), no. 2, 517-529.

[13] M. Dehghan, Implicit locally one-dimensional methods for two-dimensional diffusion with a nonlocal boundary condition, Math. Comput. Simulation 49 (1999), no. 4-5, 331-349.

[14] _ A finite difference method for a non-local boundary value problem for two-dimensional heat equation, Appl. Math. Comput. 112 (2000), no. 1, 133-142.

[15] - Fully explicit finite-difference methods for two-dimensional diffusion with an integral condition, Nonlinear Anal. Ser. A: Theory Methods 48 (2002), no. 5, 637-650.

[16] D. G. Gordeziani and G. A. Avalishvili, Solution of nonlocal problems for one-dimensional oscillations of a medium, Mat. Model. 12 (2000), no. 1, 94-103 (Russian).

[17] N. I. Ionkin, The solution of a certain boundary value problem of the theory of heat conduction with a nonclassical boundary condition, Differ. Uravn. 13 (1977), no. 2, 294-304 (Russian).

[18] J. Kačur, Method of Rothe in Evolution Equations, Teubner-Texte zur Mathematik, vol. 80, BSB B. G. Teubner Verlagsgesellschaft, Leipzig, 1985.

[19] A. Kufner, O. John, and S. Fučík, Function Spaces, Noordhoff International Publishing, Leyden, 1977.

[20] N. Merazga and A. Bouziani, Rothe method for a mixed problem with an integral condition for the two-dimensional diffusion equation, Abstr. Appl. Anal. 2003 (2003), no. 16, 899-922.

[21] C. V. Pao, Dynamics of reaction-diffusion equations with nonlocal boundary conditions, Quart. Appl. Math. 53 (1995), no. 1, 173-186.

[22] Asymptotic behavior of solutions of reaction-diffusion equations with nonlocal boundary conditions, J. Comput. Appl. Math. 88 (1998), no. 1, 225-238.

[23] Numerical solutions of reaction-diffusion equations with nonlocal boundary conditions, J. Comput. Appl. Math. 136 (2001), no. 1-2, 227-243. 
[24] L. S. Pulkina, A non-local problem with integral conditions for hyperbolic equations, Electron. J. Differential Equations 1999 (1999), no. 45, 1-6.

[25] On the solvability in $L_{2}$ of a nonlocal problem with integral conditions for a hyperbolic equation, Differ. Equ. 36 (2000), no. 2, 316-318.

[26] N. I. Yurchuk, A mixed problem with an integral condition for some parabolic equations, Differ. Uravn. 22 (1986), no. 12, 2117-2126 (Russian).

Abdelfatah Bouziani: Département de Mathématiques, Centre Universitaire Larbi Ben M’hidi, Oum El Bouaghi 04000, Algeria

E-mail address: af_bouziani@hotmail.com

Nabil Merazga: Département de Mathématiques, Centre Universitaire Larbi Ben M’hidi, Oum El Bouaghi 04000, Algeria

E-mail address: nabilmerazga@yahoo.com 